\title{
COX-2 expression correlates with VEGF-C and lymph node metastases in patients with head and neck squamous cell carcinoma
}

\author{
Panayiotis A Kyzas, Dimitrios Stefanou and Niki J Agnantis \\ Department of Pathology, University of Ioannina, Medical School, Ioannina, Greece
}

\begin{abstract}
Recent observations suggest an implication of the cyclooxygenase-2 (COX-2) in tumor lymphangiogenesis through an upregulation of vascular endothelial growth factor-C expression. It is unknown whether this mechanism also acts in squamous cell carcinoma of the head and neck region. We performed a retrospective study of $\mathbf{7 0}$ patients with head and neck squamous cell carcinoma in order to investigate whether COX-2 immunohistochemical expression correlates with vascular endothelial growth factor-C expression. We also examined the association of the expression of these molecules with clinicopathologic parameters (especially lymph node status) and outcome for these patients. We performed immunostaining on formalin-fixed, paraffinembedded tissue sections by the routine streptavidin-biotin peroxidase labeled procedure. Increased cyclooxygenase-2 expression was observed in 30 of the 68 tumor samples $(44 \%)$, while high vascular endothelial growth factor-C expression occurred in 26 of the 68 tumor samples (38\%). High expression of the two proteins was correlated with the presence of lymph node metastasis at the time of diagnosis, and the observed association was even stronger when there was overexpression for both the antibodies $(P<0.001)$. High expression of vascular endothelial growth factor-C, but not of COX-2 was correlated with increased mortality in patients with oral-larynx squamous cell carcinoma. When multivariate Cox regression model was applied, the presence of lymph node metastasis at the time of diagnosis, combined with overexpression of both the antibodies, was the only independent prognostic factor for mortality of these patients. Our results suggest that a lymphangiogenic pathway, in which COX-2 overexpression stimulates vascular endothelial growth factorC upregulation, probably exists in head and neck squamous cell carcinoma. Also, the predictive ability for mortality of regional lymph node metastasis can be improved with the combined evaluation of the immunohistochemical expression of these two proteins.

Modern Pathology (2005) 18, 153-160, advance online publication, 23 July 2004; doi:10.1038/modpathol.3800244
\end{abstract}

Keywords: cyclooxygenase-2; head and neck cancer; lymphangiogenesis; metastasis; survival; vascular endothelial growth factor-C

Clinical and pathological observations have shown that lymph node metastases at the time of diagnosis, is the most reliable prognostic factor for mortality or recurrence in patients with head and neck squamous cell carcinoma. ${ }^{1}$ However, to date, the mechanisms promoting the entrance of the cancer cells into the lymphatics and their dissemination to the cervical lymph nodes are poorly understood.

Lymphangiogenesis, the formation of new lymphatic vessels, has recently become a new research frontier in understanding tumor aggressiveness, progression and metastases. Today, intratumor lym-

Correspondence: Associate Professor D Stefanou, Department of Pathology, University of Ioannina, Medical School, 45110 Ioannina Greece.

E-mail: dstefan@cc.uoi.gr

Received 18 May 2004; revised and accepted 28 June 2004; published online 23 July 2004 phatic vessels can be directly identified with the use of new markers such as podoplanin, lymphatic vessel endothelial hyaluronan receptor (LYVE-1), desmoplakin, vascular endothelial growth factor receptor-3 (VEGFR-3) and homeobox gene prox- $1 .{ }^{2}$ Two members of the vascular endothelial growth factor (VEGF) family, VEGF-C and VEGF-D are considered as major lymphangiogenic factors. Previous reports suggest that the upregulation of VEGFC strongly correlates with regional lymph node metastases in thyroid, prostate, stomach, breast, lung and oral carcinomas. ${ }^{3,4}$

Cyclooxygenase (COX) is the key enzyme in prostaglandin's metabolism and so far, two isoforms of this enzyme have been characterized: COX-1 and COX-2. COX-1 is constitutively expressed in most tissues, and plays a role in regulating normal physiological function and inflammation. ${ }^{5}$ COX-2 has been reported to be expressed intensively in 
various malignancies ${ }^{6-8}$ and it is suggested that COX-2 overexpression correlates with tumor aggressiveness and poor prognosis. ${ }^{9}$ Thus, inhibition of COX-2 activity may have a therapeutic value. COX-2 is also proposed to be a regulator of tumor angiogenesis in head and neck squamous cell carcinoma. ${ }^{10,11}$ Recently, COX-2 was shown to be an upregulator of VEGF-C in lung adenocarcinoma, ${ }^{12}$ suggesting a role as a promoter of lymphangiogenesis.

In this retrospective study, we examined whether COX-2 immunohistochemical expression correlates with VEGF-C expression or with clinicopathologic parameters and especially lymph node involvement in a cohort of patients with head and neck squamous cell carcinoma.

\section{Materials and methods}

\section{Tissues and Clinical Parameters}

A total of 70 paraffin-embedded archival tissue blocks concerning head and neck squamous cell carcinoma specimens were obtained from the Department of Pathology of the General University Hospital of Ioannina. Specimens were, with regard to surgical material, obtained from the primary tumor. These patients with head and neck squamous cell carcinoma were treated in the Ear Nose Throat (ENT) Department (Director: Professor A Skevas) of the same hospital, between the years 1998-2002. All hematoxylin-eosin-stained sections were reviewed, the quality of the material was checked, and the best section from each specimen was selected. We selected a section showing central and peripheral areas of the tumor (intratumor and peritumor areas), avoiding areas with necrosis. Patients' records were reviewed, and clinicopathological characteristics as well as follow-up data were noted. TNM staging was established by IUCC system, and grading was performed according to WHO.

\section{Immunohistochemistry}

We performed immunostaining on formalin-fixed, paraffin-embedded tissue sections (one slide for each antibody per case) using the streptavidinbiotin peroxidase-labeled procedure. We used the ImmunoCruz Staining System (Santa Cruz Biotechnology) and the goat polyclonal antibodies VEGF-C (N-19: sc-7133, Santa Cruz Biotechnology, dilution 1:300), and COX-2 (M-19: sc-1747, Santa Cruz Biotechnology, dilution 1:100). Briefly, two adjacent $4-\mu$ m-thick sections from each paraffin-embedded block were cut. The sections were deparaffinized in xylene and rehydrated through graded concentrations of alcohol. Sections were heated in a microwave oven for two circles of 5 min each, in High pH Buffer (DAKO), for antigen retrieval. Endogenous peroxidase activity was blocked with $\mathrm{H}_{2} \mathrm{O}_{2}$ solution in methanol (0.01 M), for $30 \mathrm{~min}$. Nonspecific binding was blocked by incubating the sections for 30 min with serum block (Santa Cruz Biotechnology). After washing with phosphate-buffered saline (PBS) for $5 \mathrm{~min}$, primary antibodies were incubated overnight at $4^{\circ} \mathrm{C}$. Then sections were washed for 10 min with PBS. Biotinylated secondary antibody was applied for $30 \mathrm{~min}$, and sections were washed for $10 \mathrm{~min}$ in PBS. Sections were then incubated for 30 min with horseradish peroxidase (HRP)-Streptavidin complex. HRP-substrate was used for visualization of the expression of the antibodies and diaminobezidinetetrahydrochloride (DAB) was used as a chromogen. All sections were counterstained with hematoxylin.

\section{Antibody Expression}

Two observers (DS and PK) without knowledge of the clinical data performed evaluation of the staining. The percentage of the tumor cells that exhibited a positive cytoplasmic immunoreactivity was determined using a $\times 40$ objective lens. At least 1000 neoplastic cells were counted in each case. The intensity of the immunohistochemical staining was evaluated as follows: -, negative, + , focal expression $<5 \%$ of cancer cells, ++ , focal expression in $5-20 \%$ of cancer cells; and +++ , diffuse expression $>20 \%$ of cancer cells. Samples with ++ and +++ staining for COX-2 and VEGF-C, respectively, were classified as 'high expression group', and those with - and + were assigned as 'low expression group', as described previously. ${ }^{12}$

\section{Survival and Recurrence Analysis}

The cohort of our patients was divided into two groups regarding the location of the primary tumor. It is well known that squamous cell carcinoma of the lower lip is more curable than those of the oral cavity or the larynx,$^{13}$ and the major problem for this malignancy is local recurrence after surgical removal of the primary tumor. On the other hand, squamous cell carcinoma of the oral cavity and larynx are more aggressive. To date, the most reliable prognostic factor for survival of patients with oral or laryngeal squamous cell carcinoma is the presence of lymph node metastases at the time of diagnosis, but the accuracy of the prediction is far from perfect and certainly it can be improved. ${ }^{1}$ Thus, we performed survival analysis in the subgroup of patients with oral-larynx squamous cell carcinoma, and recurrence analysis in the subgroup of patients with squamous cell carcinoma of the lower lip. We also examined whether there was difference in survival rates when the subgroup of patients with oral-larynx squamous cell carcinoma was further separated to patients with oral squamous cell carcinoma and those with larynx squamous cell carcinoma. 


\section{Statistical Analysis}

Analyses were conducted in the SPSS software version 11.0. (SPSS, Inc., Chicago, IL, USA). VEGF-C and COX-2 expression were considered dichotomous variables using the cutoff value described above. For comparison between variables we used $\chi^{2}$ test. Analyses for survival and recurrence were conducted using the Kaplan-Meier method and different subgroups were compared using the logrank test. Cox regression model was used to determine the importance of different factors. All differences were considered positive if $P<0.05$. $P$-values are two-tailed.

\section{Results}

\section{Clinicopathologic Data}

The clinicopathologic characteristics of the patients enrolled in the study are listed in Table 1. For two patients, clinical information was inadequate, and therefore they were excluded from further analysis. Thus, a total of 68 patients were included in the analysis for the correlations of the antibodies expression with the clinicopathologic parameters. The median age of the patients was 67 (range 33-98) years. Of the patients, 53 were male and 15 were female. In total, 38 of the tumors were located in the lower lip, 21 in the oral cavity and nine in the larynx. A total of 20 patients $(29 \%)$ presented with lymph node metastases at the time of diagnosis. Only one of them suffered from lower lip squamous cell carcinoma.

Available follow-up data existed for 28 of the 31 patients with oral-larynx squamous cell carcinoma and for 33 of the 39 patients with lower lip squamous cell carcinoma. Patients were followed up until death or recurrence or for at least 24 months. Of the 28 patients with oral-larynx squamous, 17 were dead at the end of the follow-up period, and 10 of the 33 patients with lip squamous cell carcinoma showed local recurrence.

\section{COX-2 and VEGF-C Expression}

Increased, diffuse cytoplasmic staining for COX-2 was observed in 30 out of the 68 tumor samples (44\%-'High expression group'). The intensity of immunostaining was often strong, but the staining pattern was heterogeneous. Interestingly, intense staining was observed in acinic epithelial cells and ducts of normal minor salivary glands infiltrated by tumor cells (Figure 1). The same staining pattern was observed for VEGF-C, for which high expression was observed in 26 out of the 68 tumor samples (38\%) (Figure 2).

The correlation of the antibodies expression with clinicopathologic parameters is summarized in

Table 1 Correlation of clinicopathologic characteristics of the patients included in the study with immunohistochemistry results

\begin{tabular}{|c|c|c|c|c|c|c|c|}
\hline & & \multicolumn{3}{|c|}{$C O X-2$ expression } & \multicolumn{3}{|c|}{ VEGF-C expression } \\
\hline & & High & Low & $\mathrm{P}$-value & High & Low & $\mathrm{P}$-value \\
\hline \multicolumn{8}{|l|}{ Gender } \\
\hline Female & $15(24 \%)$ & 6 & 9 & NS & 3 & 12 & NS \\
\hline Male & $53(76 \%)$ & 24 & 29 & & 23 & 30 & \\
\hline \multicolumn{8}{|l|}{ Histological Grade } \\
\hline Poorly & $12(18 \%)$ & 9 & 3 & & 8 & 4 & NS \\
\hline Moderate & $24(34 \%)$ & 10 & 14 & NS $(P=0.55)$ & 8 & 16 & \\
\hline Well & $32(48 \%)$ & 11 & 21 & & 10 & 22 & \\
\hline \multicolumn{8}{|l|}{ Stage ${ }^{\mathrm{a}}$} \\
\hline I & $28(42 \%)$ & 8 & 20 & 0.035 & 2 & 26 & $<0.001$ \\
\hline II & $16(24 \%)$ & 8 & 8 & & 9 & 7 & \\
\hline III & $9(12 \%)$ & 3 & 6 & & 4 & 5 & \\
\hline IV & $15(22 \%)$ & 11 & 4 & & 11 & 4 & \\
\hline \multicolumn{8}{|l|}{ N Status } \\
\hline $\mathrm{N}$ negative (No, Nx) & $48(72 \%)$ & 16 & 32 & 0.006 & 11 & 37 & $<0.001$ \\
\hline $\mathrm{N}$ positive (N1, N2, N3) & $20(28 \%)$ & 14 & 6 & & 15 & 5 & \\
\hline \multicolumn{8}{|l|}{ Location } \\
\hline Oral & $21(31 \%)$ & 12 & 9 & 0.038 & 13 & 8 & 0.003 \\
\hline Lower lip & $38(57 \%)$ & 12 & 26 & & 8 & 30 & \\
\hline Larynx & $9(12 \%)$ & 6 & 3 & & 5 & 4 & \\
\hline \multicolumn{8}{|l|}{ COX-2 expression } \\
\hline & High & & & & 22 & 8 & $<0.001$ \\
\hline & Low & & & & 4 & 36 & \\
\hline
\end{tabular}

\footnotetext{
${ }^{\mathrm{a} A c c o r d i n g ~ t o ~ I U C C . ~}$
} 

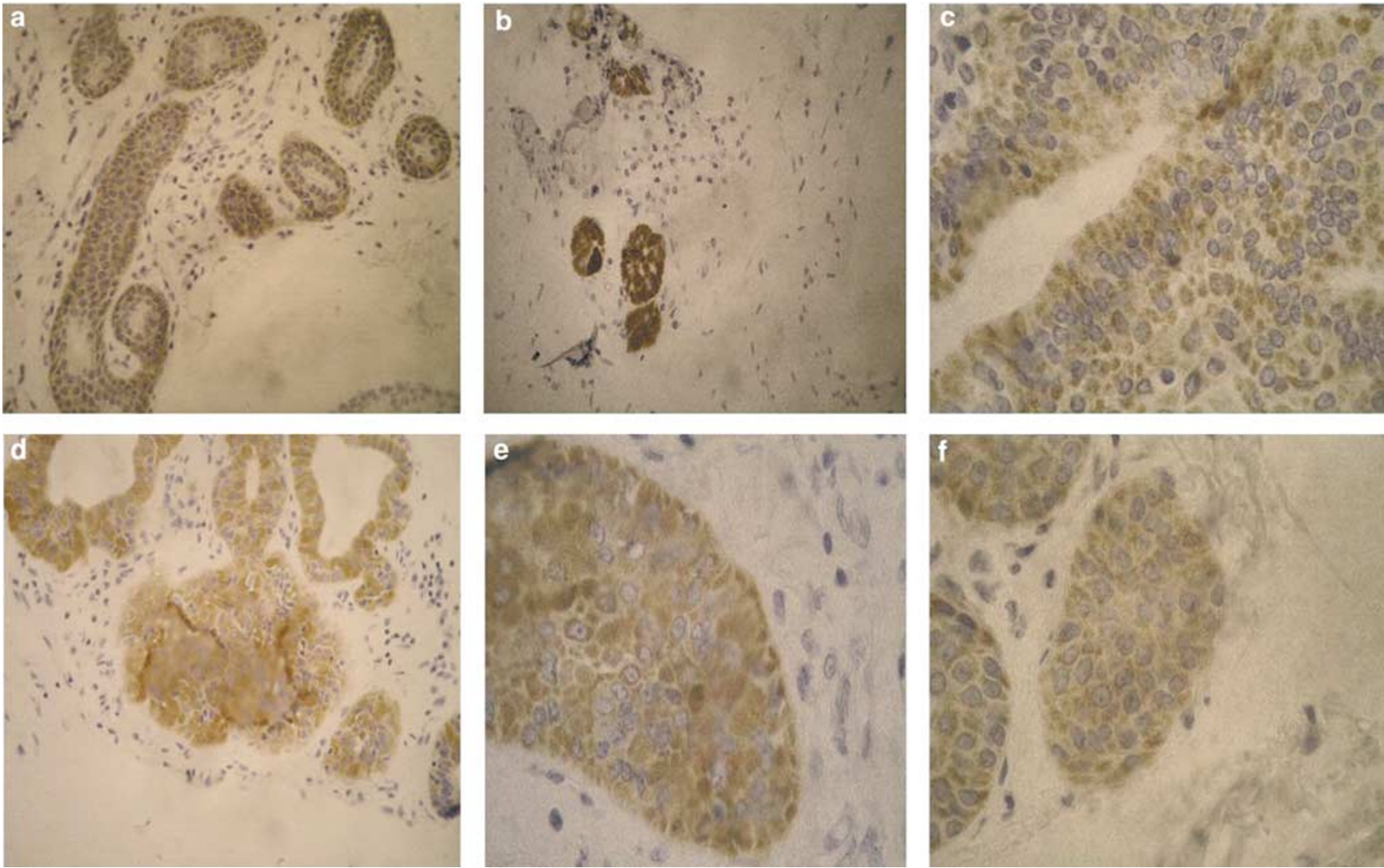

Figure 1 Diffuse cytoplasmic COX-2 immunoreactivity: in ductal epithelial cells of normal minor salivary glands (a,b magnification $\times 400$ ), in carcinoma cells in squamous cell carcinoma of the floor of the mouth, of the tongue and the larynx (c,e,f magnification $\times 1000$ ), and in both minor salivary glands and carcinoma cells in squamous cell carcinoma of the floor of the mouth (d magnification $\times 400)$.

Table 1. Increased COX-2 expression was strongly associated with the presence of lymph node metastases at the time of diagnosis $(P=0.006)$. There was also a correlation of positive COX-2 expression with higher clinical stage $(P=0.035)$. High COX-2 expression was rare in tumors located in the lower lip, whereas tumors located in the oral cavity and larynx more often expressed high COX-2 immunostaining $(P=0.038)$. No correlation was observed between COX-2 expression and histological grade, although a trend towards an association of high COX-2 expression with lower grade of differentiation could not be ruled out $(P=0.055)$. A very strong correlation was observed between COX-2 and VEGF-C expression: Out of the 30 tumor samples, which were positive for COX-2 expression, 22 also demonstrated high VEGF-C expression $(P<0.001)$.

Positive VEGF-C expression was also strongly correlated with lymph node status $(P<0.001)$ and advanced clinical stage $(P<0.001)$. VEGF-C expression was positive more often in squamous cell carcinoma of the oral cavity and larynx $(P=0.003)$. No statistically significant correlation was observed between VEGF-C expression and histological grade.

We considered the subgroup of the 22 tumors with high expression both for COX-2 and VEGF-C. A comparison between this group of patients and the group of the rest of the patients showed a strong correlation of the overexpression of both the antibodies only with the presence of lymph node metastases at the time of diagnosis $(P<0.001)$.

\section{Survival and Recurrence Analysis}

VEGF-C expression was the only prognostic factor for outcome in the subgroup of patients with oral and larynx squamous cell carcinoma (log rank $P=0.035$ ) (Figure 3). The mean survival time for VEGF-C positive expression was 21 months, while the mean survival time for VEGF-C negative expression was 42 months. Neither COX-2-positive expression nor overexpression of both antibodies had any significant association with survival. Clinical stage and lymph node status did not show any significant association with survival, although a trend towards correlation of the presence of lymph nodes at the time of diagnosis with worse survival could not be excluded (log rank $P=0.08$ ). We performed analysis for survival between the group of patients with lymph node metastases at the time of diagnosis, for which staining for both the antibodies was high (Node + /COX-2 + /VEGF-C + ), and the rest of the patients. This group of patients 

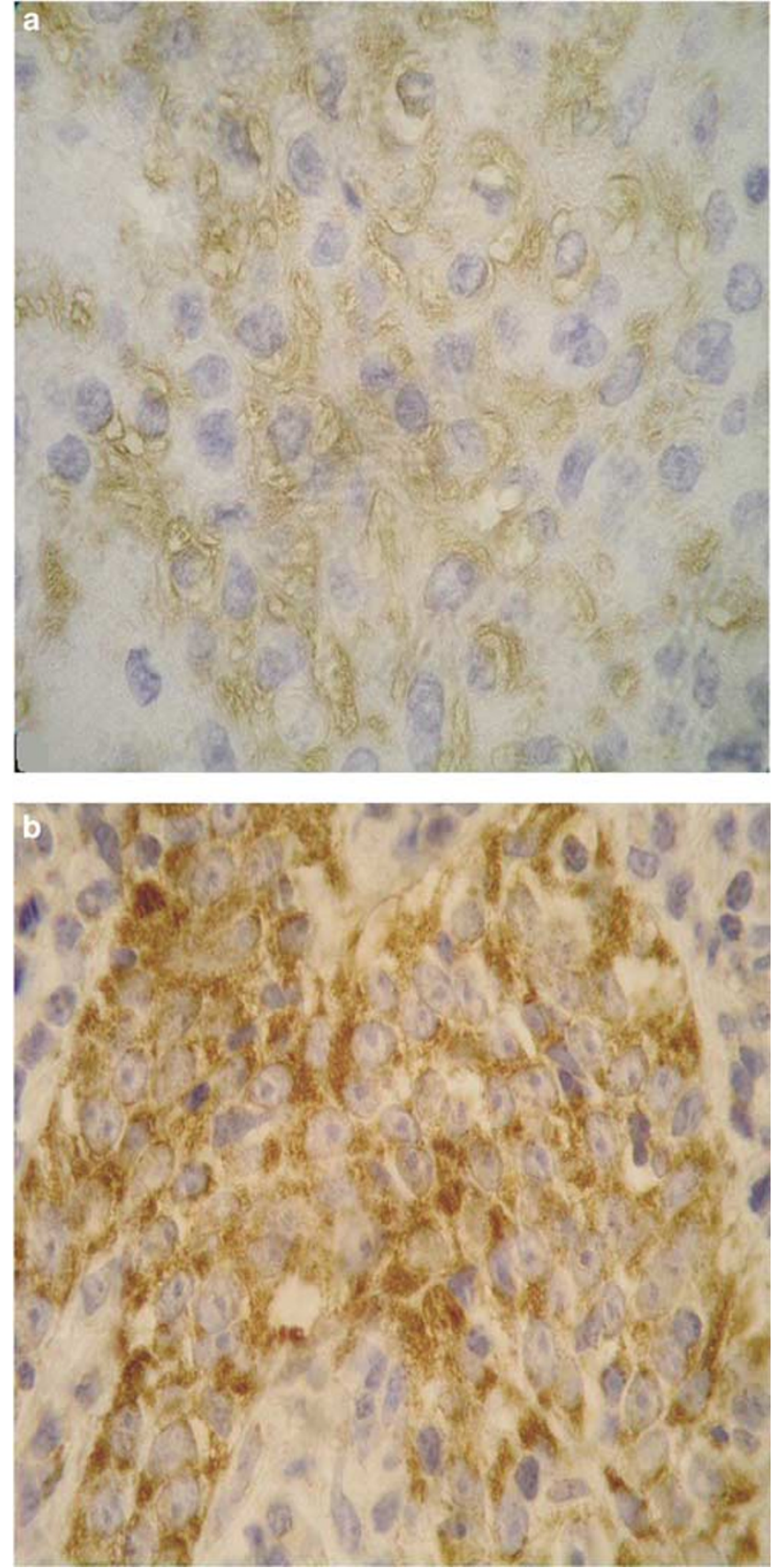

Figure 2 High VEGF-C immunoreactivity in carcinoma cells in a squamous cell carcinoma of $(\mathbf{a})$ the tongue (magnification $\times 1000$ ) and (b) larynx (magnification $\times 1000)$.

had significantly higher risk of death than the others (Figure 4 , $\log$ rank $P=0.0026$ ). Multivariate analysis with Cox regression model for the two, above described, prognostic factors, revealed that the combination of positive lymph node status with overexpression of both COX-2 and VEGF-C was the only independent prognostic factor of outcome for these patients $(P=0.006)$.

We also performed the above analyses separately for patients with oral or larynx squamous cell carcinoma. VEGF-C overexpression was correlated

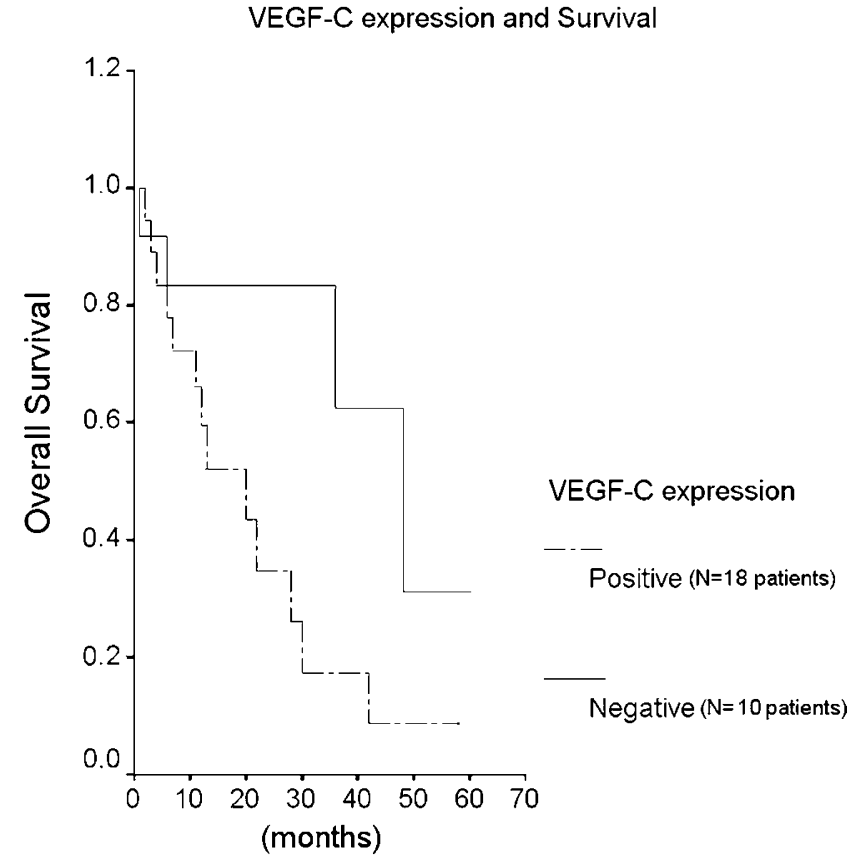

Figure 3 Kaplan-Meier curve for the overall survival of the patients with oral-larynx squamous cell carcinoma, according to the expression (VEGF-C). The two curves are significantly different (log rank $P=0.035$ )

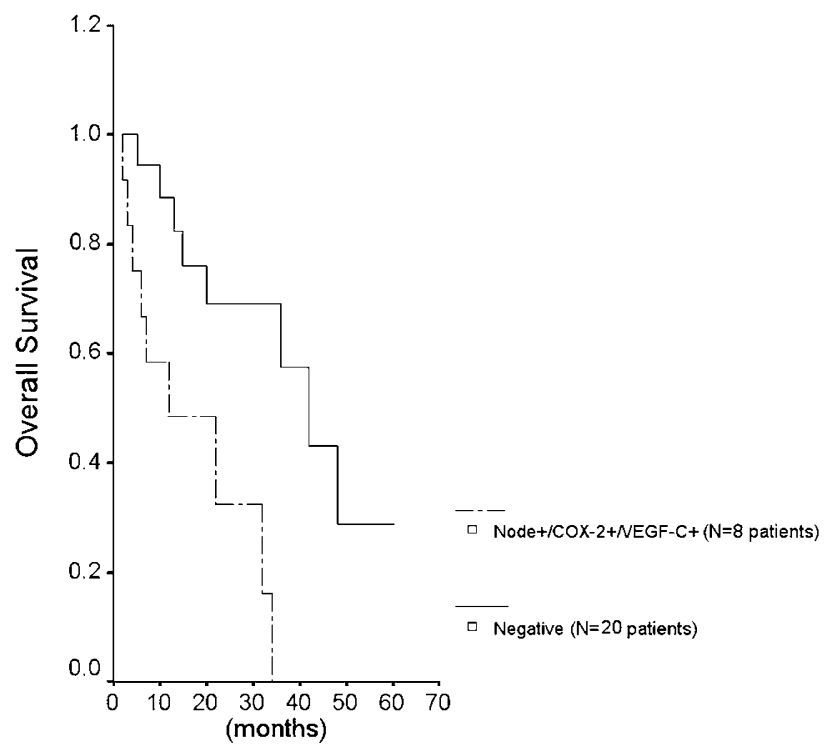

Figure 4 Kaplan-Meier curve for the overall survival of the patients with oral-larynx squamous cell carcinoma, according to lymph node status, the expression of COX-2 and the expression of (VEGF-C). COX-2 + and VEGF-C + are referred to as 'high expression groups' (see Materials and methods for more). The two curves are significantly different $(\log$ rank $P=0.0026)$.

with worse survival both in the patients with oral (log rank $P=0.02$ ) or larynx (log rank $P=0.04$ ) squamous cell carcinoma. Neither COX-2-positive expression nor overexpression of both the antibodies had any significant association with survival in both of the subgroups. Patients with oral 
squamous cell carcinoma with lymph node metastases and overexpression of COX-2 and VEGF-C (Node + /COX-2 + /VEGF-C + ), presented worse survival than the rest of the patients with oral squamous cell carcinoma (log rank $P=0.03$ ). The same pattern was observed in patients with laryngeal squamous cell carcinoma (log rank $P=0.02$ ).

Neither COX-2 nor VEGF-C overexpression showed any significant association with local recurrence for patients with squamous cell carcinoma of the lower lip. The lack of correlation remained when patients with overexpression of both the antibodies were considered as a different subgroup.

\section{Discussion}

Previous reports revealed the implication of COX-2 in squamous epithelial carcinogenesis and addressed the contribution of this enzyme to the growth and progression of head and neck squamous cell carcinoma, by various pathways. ${ }^{10,11,13,14}$ Its role is mediated by a number of molecules such as VEGF-A, CD44 and matrix metalloproteinases,${ }^{15}$ and results in the induction of tumor angiogenesis, increased invasiveness, apoptosis and immune surveillance inhibition and increased cell proliferation. Also, increased COX-2 expression was associated with poor prognosis in a number of malignancies, ${ }^{16-18}$ and head and neck squamous cell carcinoma, although the small number of patients enrolled in the studies for head and neck squamous cell carcinoma does not permit the extraction of valid conclusions. ${ }^{10,11,13,14}$

VEGF-C is a member of the VEGF family and along with VEGF-D is considered to be responsible for tumor lymphangiogenesis and lymph node metastases. ${ }^{19}$ This function is mediated via VEGF receptor-3 (VEGFR-3), which is strictly expressed only in the lymphatic vessels and stimulated by the above molecules. Recent studies suggest a direct correlation of VEGF-C expression with the presence of lymph node metastases in head and neck squamous cell carcinoma, ${ }^{20}$ and with lymphatic vessel density (LVD) in this malignancy. ${ }^{21}$ Also, other reports implied the prognostic significance of the positive expression of this protein for the clinical outcome. ${ }^{22-24}$

To our knowledge, this is the first study examining the correlation of COX-2 with VEGF-C in patients with head and neck squamous cell carcinoma. The implication of COX-2 to lymphangiogenesis is possible, since COX-2 is a pleiotropic enzyme that mediates many cellular functions. ${ }^{25}$ An upregulation of VEGF-C by COX-2 through a HER-2/Neudepentent pathway was recently described for lung adenocarcinoma. ${ }^{12}$ In this cascade, COX-2 catalyzes the synthesis of prostaglandin E2 (PGE2) that binds to EP1 prostaglandin receptor. The activation of this receptor increases the tyrosine phosphorylation of the human epidermal growth factor receptor HER/
Neu-2. Signaling is mediated through mitogenactivated protein kinase (MAPK) p38 and leads to a stimulation of the transcription factor $\mathrm{NF}-\kappa \mathrm{B}$, and finally to an increased transcription of VEGF-C gene and thus, an upregulation of this protein expression. ${ }^{26}$ It is also proposed that the mechanisms by which COX-2 and its derived prostaglandins regulate angiogenesis and lymphangiogenesis are quite distinct, since COX-2 function in angiogenesis is mediated mainly through EP $2 .{ }^{26}$ However, interactions and crosstalks between these pathways cannot be excluded. It is unknown whether the same pathway acts in squamous cell carcinoma and what are the possible crossacts (if any) with the COX-2 mediated angiogenesis procedure, for the significance of which in head and neck squamous cell carcinoma, enough evidence exists. ${ }^{10,11,27}$

In this study the most impressive finding was the strong association of the spontaneous overexpression of COX-2 and VEGF-C with the presence of lymph node metastases at the time of diagnosis. These results confirm the previous observations for each protein independently, and also suggest that a lymphangiogenic pathway, in which COX-2 overexpression stimulates VEGF-C upregulation and thus the formation of new lymphatic vessels, which is the first step for spreading and establishment of tumor cells to the regional lymph nodes, probably exists in head and neck squamous cell carcinoma. Also, in our cohort of patients, lymph node metastasis was not correlated with increased mortality. However, patients with lymph node metastases had a significantly higher risk of death when the staining was positive for both of the antibodies, than the rest of the study population. In our study, all of the eight node-positive patients with orallarynx squamous cell carcinoma, with COX-2 and VEGF-C overexpression died before 36 months. Therefore, the accuracy of the predictive ability for mortality of regional lymph node metastases can be improved with the combined evaluation of lymph node status along with the examination of the immunohistochemical expression of COX-2 and VEGF-C.

The lack of any association of both COX-2 and VEGF-C with local recurrence in squamous cell carcinoma of the lower lip is a challenging finding. In our previous work, ${ }^{28}$ we found that the immunohistochemical expression of VEGF-A was the only independent prognostic factor for recurrence for this cohort of patients. VEGF-A upregulation can be promoted not only by COX-2 pathway but also by intratumoral hypoxic conditions mediated via hypoxia-induced factor (HIF) ${ }^{29,30}$ On the other hand, as our results show, COX-2 and VEGF-C-positive expression correlate with a higher clinical stage and the presence of lymph node metastases, while the majority of these patients presented with an early stage of the disease. In fact, only one patient had lymph node metastases at the time of diagnosis. These aspects suggest that lymphangiogenesis may 
not be an early step in squamous carcinoma progression.

In conclusion, this study suggests that COX-2 correlates with VEGF-C and both of them with the presence of lymph node metastases in head and neck squamous cell carcinoma. The expression of these proteins, combined with the lymph node status of the patient, produces a promising prognostic marker for mortality of these patients. However, the complex phenomenon of tumor lymphangiogenesis in head and neck squamous cell carcinoma needs further investigation. COX-2 and VEGF-C expression should be examined in the context of other proposed lymphangiogenic molecules such as VEGF-D and the expression of VEGFR3 should also be under consideration.

\section{References}

1 Kowalski LP, Medina JE. Nodal metastases: predictive factors. Otolaryngol Clin North Am 1998;31: 621-637.

2 Podgrabinska S, Braun P, Velasco P, et al. Molecular characterization of lymphatic endothelial cells. Proc Natl Acad Sci USA 2002;99:16069-16074 (Epub 2002 November 22. Erratum in: Proc Natl Acad Sci USA 2003;100:4970).

3 Karpanen T, Egeblad M, Karkkainen MJ, et al. Vascular endothelial growth factor C promotes tumor lymphangiogenesis and intralymphatic tumor growth. Cancer Res 2001;61:1786-1790.

4 Kishimoto K, Sasaki A, Yoshihama Y, et al. Expression of vascular endothelial growth factor-C predicts regional lymph node metastases in early oral squamous cell carcinoma. Oral Oncol 2003;39:391-396.

5 Pairet M, Engelhardt G. Distinct isoforms (COX-1 and COX-2) of cyclooxygenase: possible physiological and therapeutic implications. Fundam Clin Pharmacol 1996;10:1-17.

6 Sano H, Kawahito Y, Wilder RL, et al. Expression of cyclooxygenase-1 and 2 in human colorectal cancer. Cancer Res 1995;55:3785-3789.

7 Zimmermann KC, Sarbia M, Weber AA, et al. Cyclooxygenase-2 expression in human esophageal carcinoma. Cancer Res 1999;59:198-204.

8 Hida T, Yatabe Y, Achiwa H, et al. Increased expression of cyclooxygenase 2 occurs frequently in human lung cancers, specifically in adenocarcinomas. Cancer Res 1998;58:3761-3764.

9 Tsujii M, Kawano S, DuBois RN. Cyclooxygenase-2 expression in human colon cancer cells increases metastatic potential. Proc Natl Acad Sci USA 1997;94:3336-3340.

10 Gallo O, Franchi A, Magnelli L, et al. Cyclooxygenase2 pathway correlates with VEGF expression in head and neck cancer: implications for tumor angiogenesis and metastases. Neoplasia 2001;3:53-61.

11 Gallo O, Massini E, Bianchi B, et al. Prognostic significance of cyclooxygenase-2 pathway and angiogenesis in head and neck squamous cell carcinoma. Hum Pathol 2000;33:708-714.

$12 \mathrm{Su} J \mathrm{~L}$, Shih JY, Yen ML, et al. Cyclooxygenase-2 induces EP1- and HER-2/Neu-dependent vascular endothelial growth factor-C up-regulation: a novel mechanism of lymphangiogenesis in lung adenocarcinoma. Cancer Res 2004;64:554-564.

13 Hong WK, Lippman SM, Itri LM, et al. Prevention of second primary tumors with isotretinoin in squamouscell carcinoma of the head and neck. N Engl J Med 1990;323:795-801.

14 Chan G, Boyle JO, Yang EK, et al. Cyclooxygenase-2 expression is up-regulated in squamous cell carcinoma of the head and neck. Cancer Res 1999;59:991-994.

15 Dohadwala M, Luo J, Zhu L, et al. Non-small cell lung cancer cyclooxygenase-2-dependent invasion is mediated by CD44. J Biol Chem 2001;276:2080920812 (Epub 2001 April 24).

16 Ma L, del Soldato P, Wallace JL. Divergent effects of new cyclooxygenase inhibitors on gastric ulcer healing: Shifting the angiogenic balance. Proc Natl Acad Sci USA 2002;99:13243-13247 (Epub 2002 September 13).

17 Elder DJ, Halton DE, Hague A, et al. Induction of apoptotic cell death in human colorectal carcinoma cell lines by a cyclooxygenase-2 (COX-2)-selective nonsteroidal anti-inflammatory drug: independence from COX-2 protein expression. Clin Cancer Res 1997;3:1679-1683

18 Ishiko O, Sumi $\mathrm{T}$, Yoshida $\mathrm{H}$, et al. Association between overexpression of cyclooxygenase-2 and suppression of apoptosis in advanced cancer of the uterine cervix after cyclic balloon-occluded arterial infusion. Oncol Rep 2001;8:1259-1263.

19 Joukov V, Pajusola K, Kaipainen A, et al. A novel vascular endothelial growth factor, VEGF-C, is a ligand for the Flt4 (VEGFR-3) and KDR (VEGFR-2) receptor tyrosine kinases. EMBO J 1996;15:290-298.

20 Beasly NJ, Prevo R, Banerji S, et al. Intratumoral lymphangiogenesis and lymph node metastases in head and neck cancer. Cancer Res 2002;62: 1315-1320.

21 Sedivy R, Beck-Mannagetta J, Haverkampf C, et al. Expression of vascular endothelial growth factor-C correlates with the lymphatic microvessel density and the nodal status in oral squamous cell cancer. J Oral Pathol Med 2003;32:455-460.

22 O-charoenrat P, Rhys-Evans P, Eccles SA. Expression of vascular endothelial growth factor family members in head and neck squamous cell carcinoma correlates with lymph node metastases. Cancer 2001;92: 556-568.

23 Neuchrist C, Erovic BM, Handisurya A, et al. Vascular endothelial growth factor $\mathrm{C}$ and vascular endothelial growth factor receptor 3 expression in squamous cell carcinomas of the head and neck. Head Neck. 2003;25:464-474.

24 Shintani S, Li C, Ishikawa $\mathrm{T}$, et al. Expression of vascular endothelial growth factor A, B, C, and D in oral squamous cell carcinoma. Oral Oncol 2004;40: 13-20.

25 Narumiya S, Sugimoto Y, Ushikubi F. Prostanoid receptors: structures, properties, and functions. Physiol Rev 1999;79:1193-1226.

26 Tsai PW, Shiah SG, Lin MT, et al. Up-regulation of vascular endothelial growth factor $\mathrm{C}$ in breast cancer cells by heregulin-beta 1 . A critical role of p38/nuclear factor-kappa B signaling pathway. J Biol Chem 2003;278:5750-5759 (Epub 2002 December 05).

27 Jaeckel EC, Raja S, Tan J, et al. Correlation of expression of cyclooxygenase-2, vascular endothelial 
growth factor, and peroxisome proliferator-activated receptor delta with head and neck squamous cell carcinoma. Arch Otolaryngol Head Neck Surg 2001; 127:1253-1259.

28 Kyzas AP, Stefanou D, Agnantis NJ. Immunohistochemical expression of vascular endothelial growth factor correlates with positive surgical margins and recurrence in T1 and T2 squamous cell carcinoma (SCC) of the lower lip. Oral Oncol (in press).

29 Mohamed KM, Le A, Duong $\mathrm{H}$, et al. Correlation between VEGF and HIF-1alpha expression in human oral squamous cell carcinoma. Exp Mol Pathol 2004;76:143-152.

30 Beasley NJ, Leek R, Alam M, et al. Hypoxia-inducible factors HIF-1alpha and HIF-2alpha in head and neck cancer: relationship to tumor biology and treatment outcome in surgically resected patients. Cancer Res 2002;62:2493-2497. 